\title{
Large eddy and direct numerical simulations of a turbulent water-filled differentially heated cavity of aspect ratio 5
}

\author{
D. Kizildaga, F.X Trias ${ }^{\mathrm{a}}$, I. Rodríguez ${ }^{\mathrm{a}}$, A. Oliva ${ }^{\mathrm{a}, *}$ \\ ${ }^{a}$ Heat and Mass Transfer Technological Center (CTTC) \\ Universitat Politècnica de Catalunya - BarcelonaTech (UPC) \\ Colom 11, 08222 Terrassa, Barcelona, Spain
}

\begin{abstract}
Natural convection in a differentially heated cavity is characterized by different phenomena such as laminar to turbulent flow transition in the boundary layer, turbulent mixing, and thermal stratification in the core of the cavity. In order to predict the thermal and fluid dynamic behavior of the flow in these cavities, the location of transition to turbulence should be accurately determined. In this work, the performance of three subgrid-scale (SGS) models is submitted to investigation in a water-filled cavity of aspect ratio 5 at Rayleigh number $R a=3 \times 10^{11}$. To do so, the models are compared with the solution obtained by means of direct numerical simulation. The models tested are: (i) the wall-adapting local-eddy viscosity (WALE) model, (ii) the QR model, (iii) the WALE model within a variational multiscale framework (VMS-WALE). It has been shown that the VMS-WALE and WALE models perform better in estimating the location of transition to turbulence, and
\end{abstract}

\footnotetext{
*Corresponding author; Tel:+34 9373981 92; Fax: +34 9373989 20; e-mail: cttc@cttc.upc.edu (A. Oliva)
} 
thus their overall behavior is more accurate than the QR model. The results have also revealed that the use of SGS models is justified in this flow as the transition location and consequently the flow structure cannot be captured properly if no model is used for the tested spatial resolution.

Keywords: LES, DNS, Turbulence, Differentially heated cavity, Natural convection

\section{Introduction}

The flow in a differentially heated cavity (DHC) has been studied extensively in the literature, due to its relevance to model many applications of industrial interest, like air flow in buildings, heat transfer in solar collectors, or cooling of electronic devices. The flow is characterized by a thermal gradient which is orthogonal to the gravitational field. In accordance with the available computing power, first efforts mainly addressed steady laminar two-dimensional flows (see for instance [1]). In the last two decades, more demanding two- and three-dimensional transitional and turbulent flows appeared in the literature thanks to the increasing computational resources, which allowed dealing with different scales of motion present in such flows at moderate and high Rayleigh numbers $(R a)$ [2-5]. Although the geometry is rather simple, different regimes coexist when the Rayleigh number is increased beyond a critical value: i) laminar flow in the upstream part of the vertical boundary layers and in the core of the cavity, ii) transitional and turbulent flow at some location where the downstream traveling waves grow sufficiently to disrupt the vertical boundary layer and eject large unsteady eddies. In the core of the cavity, a thermally stratified zone is expected. Even 
though at relatively smaller velocities compared with the vertical boundary layer, the core is in motion, as the isotherms oscillate around a mean horizontal profile, which can be attributed to the internal gravity waves. Although conducting direct numerical simulation (DNS) in real-scale engineering problems is not feasible yet, the present configuration, being a canonical case, has been subject of DNS studies on moderate Rayleigh numbers [6-8]. These works have contributed to understand the physical phenomena of turbulence and provided useful data.

The vast majority of the performed studies consider air-filled cavities (Prandtl number $\operatorname{Pr} \approx 0.7$ ). However, the working fluid is water for many buoyancy-driven applications. In the case of a water-filled cavity, i.e. greater Prandtl number compared with air, obtaining solutions for the governing equations gets more complicated since the thermal boundary layer becomes thinner for the same Rayleigh number [9]. Therefore, finer grids are required to capture the smallest scales of the flow. This may be one of the reasons explaining that numerical studies of water-filled DHC are quite scarce when compared with air-filled ones and are generally limited to a two-dimensional domain [10-12]. Alternatively, large eddy simulations (LES) are an encouraging alternative for the resolution of turbulent natural convection in these cavities as they model the smallest scales of flow while the large scales are solved. The selection of an appropriate subgrid-scale (SGS) stresses model for describing the complex flow behavior is crucial in this cavity flow, where the overall performance of the model is dependent on the accurate prediction of the transition to turbulence phenomenon.

With regard to the use of LES models in DHC flows, several authors pub- 
lished relevant contributions for the air-filled cavities. Peng and Davidson [13] studied a flow with a low level of turbulence $\left(R a=1.58 \times 10^{9}\right)$ in a cubical cavity. They obtained good results in the main flow quantities by using approximately 0.6 million control volumes $(\mathrm{CV})$. On the other hand, second-order turbulent statistics showed discrepancies with the reference experimental work, due mainly to the lower resolution of the grid in the outer shear layer and the spanwise direction.

In the recent years, different studies of LES on a DHC configuration of aspect ratio 5 appeared in the literature. Barhaghi and Davidson [14] studied a turbulent DHC of aspect ratio 5 at $R a=4.028 \times 10^{8}$ (based on the cavity width) by means of the Smagorinsky [15], the dynamic eddy-viscosity [16], and the wall-adapting local-eddy viscosity (WALE) [17] models. The finest mesh they employed had approximately 2.5 million CVs. The tested SGS models gave substantially different results in capturing the location of transition in the vertical boundary layer, with the dynamic eddy viscosity model the most accurate one. They performed a full analysis of turbulent statistics in both transitional and turbulent regions of the cavity. They indicated that the results in the transition region showed considerable differences between the coarse and fine meshes, while in the fully turbulent region the grid dependency was no longer important.

More recently, two works for very similar Rayleigh numbers were carried out using the same aspect ratio. Lau et al. [18] tested different SGS models in an air-filled DHC of aspect ratio 5 at $R a=4.56 \times 10^{10}$ using approximately 2.1 million CVs. The comparison with the available experimental data demonstrated that the transition to turbulence cannot be well captured 
if the models are too dissipative, thus leading to important discrepancies with the reference data. Trias et al. [19] studied a turbulent DHC flow of aspect ratio 5 by means of DNS and regularization modeling for $R a=4.5 \times 10^{10}$. For the modeling of turbulence they used a novel class of regularization that restrain the convective production of small scales of motion in an unconditionally stable manner. The comparison of the results showed that the method was able to capture the general patterns of flow even for very coarse meshes, illustrating the potential of the regularization method to deal with complex flows.

Ghaisas et al. [20] tested different SGS models for weakly turbulent flow in a DHC of aspect ratio 4 for two different Rayleigh numbers, obtaining good agreement with the available DNS data for $R a=6.4 \times 10^{8}$, although higher mesh resolution was shown to be needed when the Rayleigh number was raised to $2.0 \times 10^{9}$.

Sergent et al. [21] recently used LES to show that the discrepancies between the numerical and experimental stratification values in the center of the air-filled DHC is due to the contribution of the front and rear end walls in heat transfer and fluid flow.

The conducted studies so far showed that the accurate assessment of the transition location is essential in predicting flow configuration in a turbulent DHC flow. As a result, the performance of the SGS models are primarily dependent on their capability to capture this location. Considering this, the present work aims at testing the performance of three SGS models for the turbulent natural convection flow in a water-filled DHC of aspect ratio 5 at $R a=3 \times 10^{11}$. The tested models are: (i) the WALE model [17], (ii) the 
QR model [22], and (iii) the WALE model within a variational multiscale framework [23] (VMS-WALE). In the literature, for the DHC configuration, the numerical investigations of LES are concentrated on the air-filled cavities. Thus, the current study is intended to fill a gap, providing DNS and LES results for moderate-to-high Rayleigh numbers in three-dimensional waterfilled cavities. Moreover, to the best knowledge of the authors, this is the first study to test the QR model in a DHC configuration.

The remainder of the present paper is organized as follows. In the next section, the description of the case is presented. Then, Section 3 is devoted to the DNS: the numerical methodology used is outlined and the details of the verification studies are explained. In Section 4, the LES models tested in the study are briefly described. In Section 5, the performance of the LES models is assessed by comparison with the DNS results. The discussion is focused on the prediction of the transition to turbulence location and its influence on the flow structure and heat transfer. Finally, concluding remarks are given in Section 6.

\section{Description of the case}

The turbulent natural convection in a three dimensional DHC of height $\mathrm{H}$, width $\mathrm{W}$, and depth $\mathrm{D}$ is submitted to investigation (see Figure 1). The aspect ratio is $H / W=5$. This aspect ratio was studied previously by different authors $[14,18,19,24]$. The cavity is subjected to heating along the left vertical wall, and cooling along the right vertical wall by means of isothermal confining walls at $T_{h}$ and $T_{c}$, respectively. The cavity height based Rayleigh number $\left(R a=g \beta\left(T_{h}-T_{c}\right) H^{3} \operatorname{Pr} / \nu^{2}\right)$ is $3 \times 10^{11}$, where $g$ is the 
gravity, $\beta$ is the thermal expansion coefficient, and $\nu$ is the kinematic viscosity. The Prandtl number $(\operatorname{Pr}=\nu / \alpha)$ in this water-filled DHC is 4.31, which corresponds to water at $40^{\circ} \mathrm{C}$, where $\alpha$ is the thermal diffusivity. The confining walls at the top and bottom of the cavity are adiabatic. No-slip velocity condition is imposed on these four boundaries in $x$ - and $y$-directions, whereas periodic boundary conditions are imposed in the $z$-direction for all the variables.

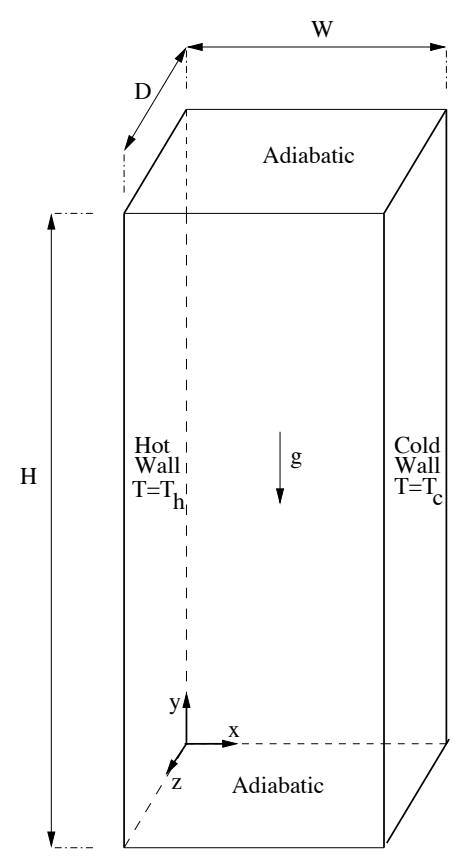

Figure 1: Schema of the three-dimensional DHC. 


\section{DNS}

\subsection{Governing equations}

Considering an incompressible viscous Newtonian fluid, assuming the Oberbeck-Boussinesq (OB) approximation, and neglecting thermal radiation, the governing equations read,

$$
\begin{aligned}
\nabla \cdot \mathbf{u} & =0 \\
\frac{\partial \mathbf{u}}{\partial t}+(\mathbf{u} \cdot \nabla) \mathbf{u}-\nu \nabla^{2} \mathbf{u}+\nabla p+\mathcal{F} & =\mathbf{0} \\
\frac{\partial T}{\partial t}+(\mathbf{u} \cdot \nabla) T-\alpha \nabla^{2} T & =0
\end{aligned}
$$

where $\mathbf{u}=(u, v, w)$ is the velocity vector, $p$ and $T$ are the kinematic pressure and temperature fields, respectively. $\mathcal{F}$ is the body force $\mathcal{F}=\beta\left(T-T_{m}\right) \mathbf{g}$, with $T_{m}=\left(T_{h}+T_{c}\right) / 2$ the mean temperature, and $\mathbf{g}=(0,-g, 0)$ the gravitational acceleration vector.

For the presentation of the dimensionless results, the adopted reference length, time, velocity, and temperature are $L_{r e f}=H, t_{r e f}=\left(H^{2} / \alpha\right) R a^{-0.5}$, $u_{\text {ref }}=(\alpha / H) R a^{0.5}$, and $\Delta T_{\text {ref }}=\left(T_{h}-T_{c}\right)$, respectively.

\subsection{Numerical methods for DNS}

The incompressible Navier-Stokes equations (1)-(2) are discretized on a staggered Cartesian grid by a fourth-order symmetry-preserving numerical scheme [25], representing the convective term by a skew-symmetric, and the diffusive term by a symmetric positive-definite matrices. The resulting operator-based finite-volume discretization of equations (1)-(2) for the temporal evolution of the spatially discrete staggered velocity vector $\mathbf{u}_{s}$ yields, 


$$
\begin{aligned}
M \mathbf{u}_{s} & =\mathbf{0}_{c} \\
\Omega \frac{d \mathbf{u}_{s}}{d t}+C\left(\mathbf{u}_{s}\right) \mathbf{u}_{s}+D \mathbf{u}_{s}-M^{T} \mathbf{p}_{c} & =\mathbf{0}_{s}
\end{aligned}
$$

where the subindices $c$ and $s$ refer to cell-centered and staggered discrete variables, respectively. The diffusive matrix, $D$, is symmetric and positive semi-definite, representing the integral of the diffusive flux $-\nu \nabla \mathbf{u} \cdot \mathbf{n}$ through the CV faces, where $\mathbf{n}$ is the unit vector normal to the CV face. The diagonal matrix, $\Omega$, stands for the sizes of the control volumes and the approximate convective flux is discretized as in [25]. The resulting convective matrix, $C\left(\mathbf{u}_{s}\right)$, is skew-symmetric. Then, in the absence of body forces, the time evolution of the discrete kinetic energy $\left\|\mathbf{u}_{s}\right\|^{2}=\mathbf{u}_{s} \cdot \Omega \mathbf{u}_{s}$ is governed by,

$$
\frac{d}{d t}\left\|\mathbf{u}_{s}\right\|^{2}=-\mathbf{u}_{s} \cdot\left(D+D^{T}\right) \mathbf{u}_{s}<0
$$

where the convective and pressure gradient contributions cancel because of the skew-symmetry of the convective term and the incompressibility constraint of Equation (4), respectively. Therefore, even for coarse grids, the energy of the resolved scales of motion is convected in a stable manner, i.e. the discrete convective operator transports energy from a resolved scale of motion to other resolved scales without introducing any artificial dissipation of energy. The cell-centered temperature is discretized in a similar manner. For details, the reader is referred to [25].

For the temporal discretization, a second-order explicit one-leg scheme [26] is used by means of the classical fractional step projection method. A predictor velocity, $\mathbf{u}_{s}^{p}$, is explicitly evaluated without considering the contribution of the pressure gradient. Then, by imposing the incompressibility constraint, 
$M \mathbf{u}_{s}^{n+1}=\mathbf{0}_{s}$, it leads to a Poisson equation for $\mathbf{p}_{c}^{n+1}$ to be solved once each time-step,

$$
-M \Omega^{-1} M^{T} \mathbf{p}_{c}^{n+1}=M \mathbf{u}_{s}^{p}
$$

For the details of the numerical algorithms and the Poisson solver, the reader is referred to $[7]$.

\subsection{Verification of the DNS}

The DNS code was verified using the Method of Manufactured Solutions (MMS) [27], and tested for several benchmark reference results (see for instance [7]). Moreover, since the spatial discretization is fully-conservative, the exact fulfillment of the global kinetic energy balance was also used as an additional verification. Regarding the simulations, sufficiently fine grid resolution in space and time is required in order to resolve all the relevant turbulent scales, whereas the periodic $z$-direction should be large enough to contain the largest scales of the flow. Furthermore, the flow statistics should be integrated for a time span long enough to evaluate them properly. Note, however, that a compromise between accuracy and the cost of the calculations has to be adopted.

In the present DNS, the flow is initially at rest with a random perturbation of the initial temperature field to trigger three-dimensionality. The flow statistics are averaged over the three statistically invariant transformations: time, spanwise direction, and central-point symmetry. This averaging is denoted as $\langle\cdot\rangle$. The total simulation period is 600 time units, where the last 200 time units are used for the flow statistics. Three meshes are used for the 


\begin{tabular}{lcccccccc}
\hline Case & $N_{x}$ & $N_{y}$ & $N_{z}$ & $N_{\text {tot }} \times 10^{-6}$ & $D / H$ & $\gamma_{x}$ & $(\Delta x)_{\min } / H$ & $\Delta t / t_{\text {ref }}$ \\
\hline Mesh A & 240 & 648 & 128 & 19.91 & 0.1 & 2.0 & $6.20 \times 10^{-5}$ & $2.71 \times 10^{-4}$ \\
Mesh B1 & 160 & 432 & 128 & 8.85 & 0.1 & 2.0 & $9.38 \times 10^{-5}$ & $8.06 \times 10^{-4}$ \\
Mesh B2 & 160 & 432 & 256 & 17.69 & 0.2 & 2.0 & $9.38 \times 10^{-5}$ & $8.06 \times 10^{-4}$ \\
\hline
\end{tabular}

Table 1: Numerical simulation parameters for DNS. $N_{x}, N_{y}$, and $N_{z}$ are the number of CVs in wall-normal, streamwise, and spanwise directions, respectively. $N_{\text {tot }}$ is the number of total CVs, $(\Delta x)_{\min }$ is the smallest wall-normal distance, $\gamma_{x}$ is the mesh concentration factor, and $\Delta t$ is the average time-step.

grid study. The numerical parameters of the simulations are given in Table 1. Grid spacing in the streamwise and spanwise directions is chosen uniform, while for the wall-normal direction, the following hyperbolic-tangent function is used:

$$
x_{i} / H=\frac{W}{2 H}\left(1+\frac{\tanh \left[\gamma_{x}\left(2(i-1) / N_{x}-1\right)\right]}{\tanh \gamma_{x}}\right) \quad i=1,2, \ldots, N_{x}+1
$$

where $x_{i}$ indicates the location of the control volume face, $\gamma_{x}$ is the mesh concentration factor and $N_{x}$ is the number of CVs in the wall-normal direction.

Spanwise two-point correlation, $R_{\phi \phi}$, defined as

$$
R_{\phi \phi}(x, y, \delta z)=\frac{<\phi^{\prime}(x, y, z) \phi^{\prime}(x, y, z+\delta z)>}{<\phi^{\prime 2}(x, y, z)>}
$$

with $\phi^{\prime}$ the fluctuation of the variable $\phi\left(\phi^{\prime}=\phi-<\phi>\right)$, is used to check the adequacy of the computational domain in the periodic direction. In this regard, two meshes, Mesh B1 and Mesh B2, are considered. Note that these grids only differ in the length of the domain in the periodic direction, as Mesh 
B2 has twice larger domain than Mesh B1. In Figure 2, the correlation values are plotted using the spanwise velocity component, $w$, at six monitoring points for these meshes. In all the monitoring points, the correlations fall off to approximately zero at separations of one half-period even for the narrower Mesh B1, showing that this computational domain length in the spanwise direction $(D / H=0.1)$ is sufficiently large. It should be pointed out that, for the narrower mesh, the correlations drop only approximately to zero for some monitoring points. However, given the similarity of the two-point correlation values for both meshes, the choice of the narrower domain is only expected to alter the size of the largest structures in a small degree, which in turn should not affect the first- and second-order statistics significantly, as was shown in [28]. Although not shown here, similar results are obtained for other monitoring points and variables.
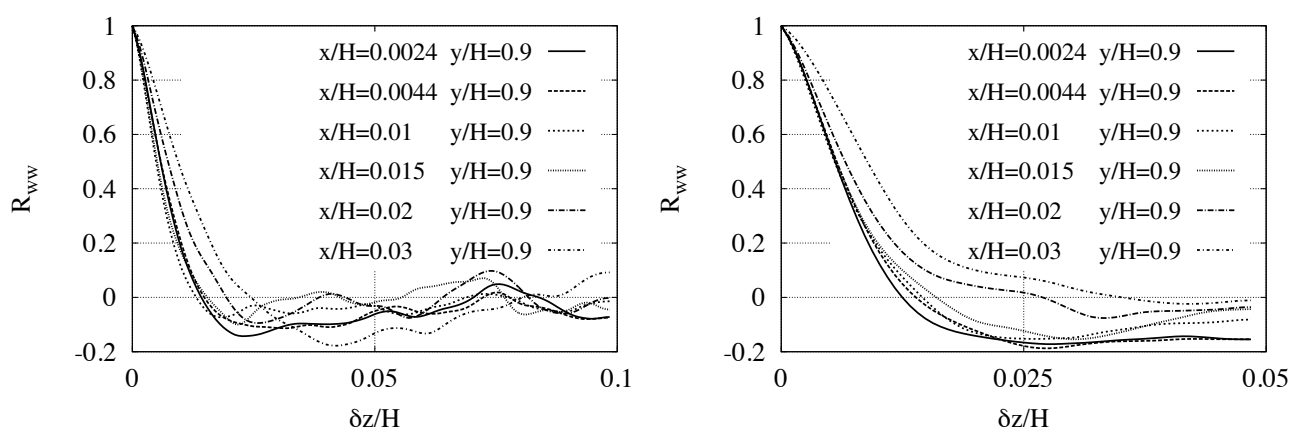

Figure 2: Two-point correlations of the spanwise velocity, $w$, at six monitoring locations for Mesh B2 (left) and Mesh B1 (right).

When constructing the mesh, the proper resolution of the boundary layer should be guaranteed. Based on the periodic direction domain size of Mesh B1, Mesh A improves considerably the grid resolution in the wall- 
normal and streamwise directions. Figure 3 displays the location of the first grid point in wall-units, $(\Delta x)^{+}=u_{\tau}(\Delta x)_{\min } / \nu$, for Mesh A, where $u_{\tau}$ is the friction velocity, $u_{\tau}=\left(\tau_{w} / \rho\right)^{1 / 2}$, and $\tau_{w}$ is the wall friction, $\tau_{w}=\rho \nu \partial v / \partial x$. Note that $(\Delta x)^{+}$falls down to values smaller than unity, indicating sufficient resolution in the wall-normal direction.

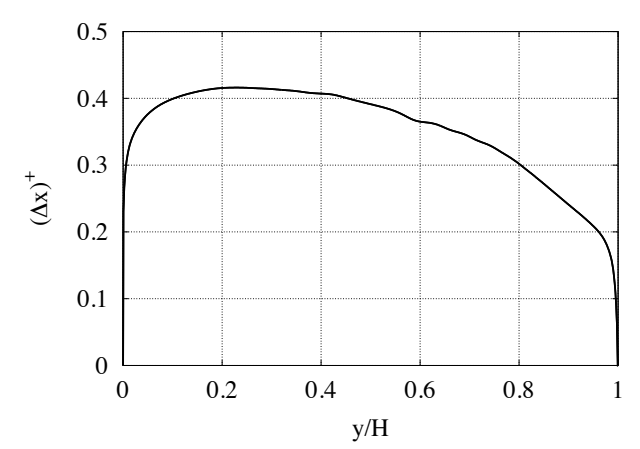

Figure 3: Location of the first grid point in wall-units for Mesh A.

In order to ensure that the smallest relevant scales are well-resolved, the grid resolution in the spanwise direction has to be adjusted. To that end, one-dimensional energy spectra of the spanwise velocity component, $E_{w w}\left(x, y, k_{z}\right)=\hat{w}_{k_{z}}(x, y) \hat{w}_{k_{z}}^{*}(x, y)$, at six different monitoring points have been checked for the suitability of the numerical simulation parameters in the $z$-direction using Mesh A, where $(\cdot)^{*}$ represents the complex conjugate. As can be seen in Figure 4, there is no energy pile-up at high wavenumbers, and the magnitude of the energy density between the smallest and the largest wavenumbers has dropped several orders of magnitude, which indicates that the grid is fine enough to resolve the smallest relevant scales.

Finally, in support of the present analysis, the local average Nusselt num- 

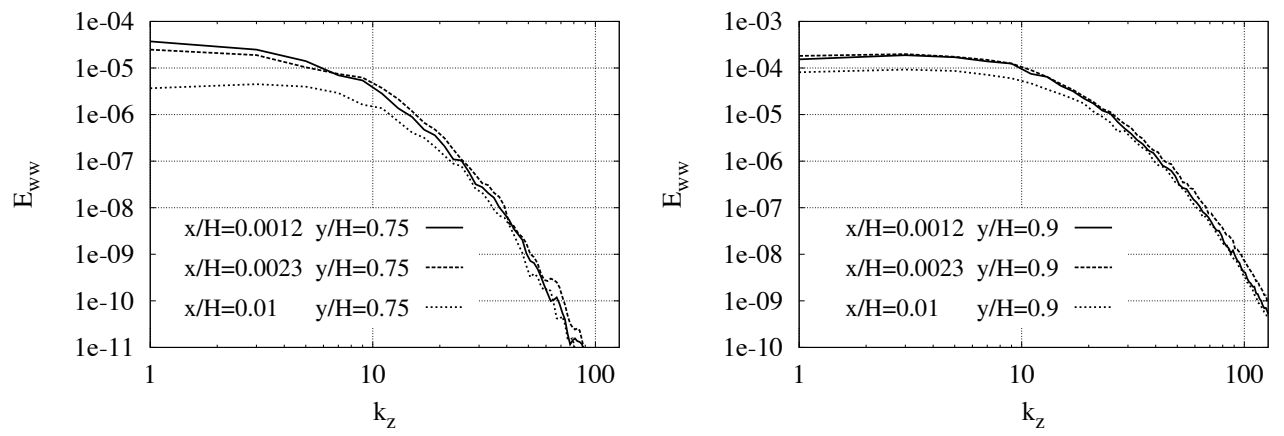

Figure 4: Energy spectra of the spanwise velocity component at six monitoring locations for Mesh A.

ber, defined as

$$
<N u>=-\left.\frac{H}{\Delta T_{r e f}} \frac{\partial<T>}{\partial x}\right|_{x=0}
$$

for a given position at the hot wall is depicted together with the profile of temperature fluctuations, $\left\langle T^{\prime} T^{\prime}\right\rangle / \Delta T_{\text {ref }}^{2}$, in Figure 5. In agreement with the two-point correlations, the results are only slightly affected by the domain size as Mesh B1 and Mesh B2 are concerned, thus confirming that the chosen spanwise domain size is adequate. It must be borne in mind that, a reasonable trade-off between accuracy and CPU-time is necessarily adopted, given the cost of the present calculations.

Thus, considering the present analysis, the numerical results obtained with Mesh A will be hereafter referred to as the DNS solution. 

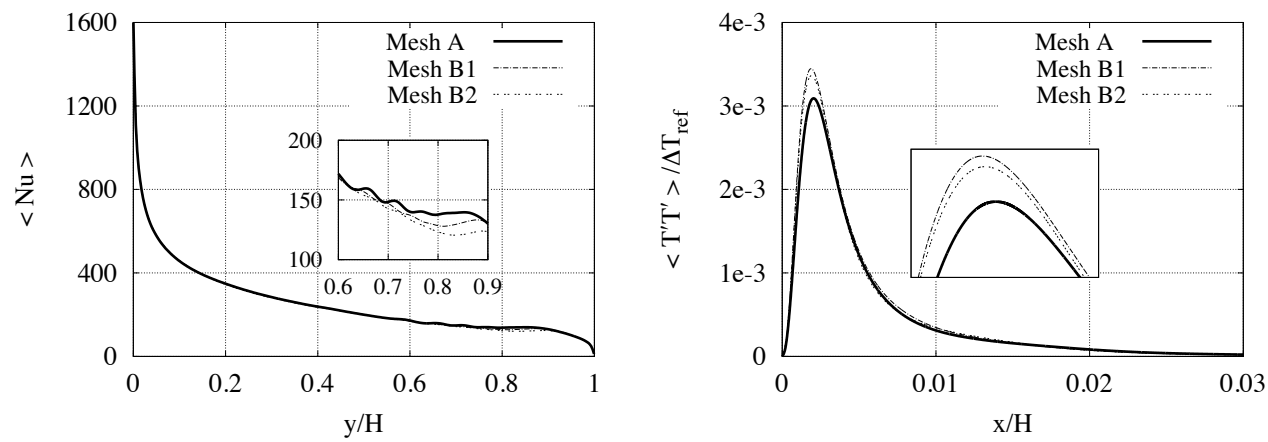

Figure 5: Local average Nusselt number (left) and temperature fluctuations, $\left\langle T^{\prime} T^{\prime}\right\rangle$ $/ \Delta T_{r e f}^{2}$, in the vicinity of the hot wall at $y / H=0.9$ (right) for the tested meshes.

\section{LES}

\subsection{Governing equations}

LES equations are obtained by means of spatially filtering the Equations 1-3:

$$
\begin{aligned}
\nabla \cdot \overline{\mathbf{u}} & =0 \\
\frac{\partial \overline{\mathbf{u}}}{\partial t}+(\overline{\mathbf{u}} \cdot \nabla) \overline{\mathbf{u}}-\nu \nabla^{2} \overline{\mathbf{u}}+\nabla \bar{p}+\overline{\mathcal{F}} & =-\nabla \cdot \mathcal{T} \\
\frac{\partial \bar{T}}{\partial t}+(\overline{\mathbf{u}} \cdot \nabla) \bar{T}-\alpha \nabla^{2} \bar{T} & =-\nabla \cdot \mathcal{T}_{T}
\end{aligned}
$$

where $\overline{(\cdot)}$ represents a linear spatial filter that commutes with the differential operators. The last terms in Equations (12) and (13) indicate some modeling of the filtered non-linear convective term. Due to its simplicity and robustness in complex configurations, the present work considers eddy-viscosity models (see [29], for instance). In this case, the traceless part of the SGS stress tensor, $\mathcal{T}$, is defined as,

$$
\mathcal{T}-\frac{1}{3}(\mathcal{T}: \boldsymbol{I}) \boldsymbol{I}=-2 \nu_{\text {sgs }} \overline{\mathcal{S}}
$$


where $\overline{\mathcal{S}}$ is the rate-of-strain tensor, $\overline{\mathcal{S}}=\frac{1}{2}\left[\nabla(\overline{\mathbf{u}})+\nabla^{*}(\overline{\mathbf{u}})\right]$, and $\nabla^{*}$ is the transpose of the gradient operator. $\mathcal{T}_{T}$ term is evaluated like the $\mathcal{T}$ term, but $\nu_{s g s}$ is substituted by $\nu_{s g s} / P r_{t}$, where $P r_{t}$ is the turbulent Prandtl number (0.4 in this work).

To close the formulation, a suitable expression for the SGS viscosity, $\nu_{s g s}$, must be introduced. In this work, the behavior of different models have been investigated: (i) the WALE model [17], (ii) the QR model [22], (iii) the VMS-WALE model [23]. A brief description of these models is given below.

\subsubsection{Wall-adapting local-eddy viscosity (WALE) model}

This model is based on the square of the velocity gradient tensor, and it accounts for the effects of both the strain and the rotation rate of the smallest resolved turbulent fluctuations. It was formulated with the following properties: i) vanishing eddy-viscosity $\left(\nu_{s g s}=0\right)$ in the regions where the flow is laminar; ii) for any two-dimensional flow $\nu_{s g s}=0$; iii) at no-slip walls eddy-viscosity also vanishes as $\nu_{s g s} \propto y^{3}$ and, iv) eddy-viscosity vanishes as the filter size $(\ell)$ approaches the smallest scales of the flow (i.e. $\nu_{s g s} \rightarrow 0$ when $\left.\ell \propto R e^{-3 / 4}\right)$. The model reads,

$$
\begin{aligned}
\nu_{s g s} & =\left(C_{w} \ell\right)^{2} \frac{(\overline{\mathcal{V}}: \overline{\mathcal{V}})^{\frac{3}{2}}}{(\overline{\mathcal{S}}: \overline{\mathcal{S}})^{\frac{5}{2}}+(\overline{\mathcal{V}}: \overline{\mathcal{V}})^{\frac{5}{4}}} \\
\overline{\mathcal{V}} & =\frac{1}{2}\left[\nabla(\overline{\mathbf{u}})^{2}+\nabla^{*}(\overline{\mathbf{u}})^{2}\right]-\frac{1}{3}\left(\nabla(\overline{\mathbf{u}})^{2}: \boldsymbol{I}\right) \boldsymbol{I}
\end{aligned}
$$

where $C_{w}$ is the model constant, whose value lies in the range between 0.3 and 0.5. In the present calculations, a value of $C_{w}=0.325$ is used. The filter length, $\ell$, is evaluated as the cube root of the volume of the cell. 


\subsubsection{QR eddy-viscosity model}

This model has similar properties as the WALE model except for the linear dependance of $\nu_{s g s}$, i.e. $\nu_{s g s} \propto y$, and it is based on the invariants of the rate-of-strain tensor. The model reads,

$$
\nu_{s g s}=\left(C_{q r} \ell\right)^{2} \frac{r^{+}}{q}
$$

where $C_{q r}=1 / \pi+1 / 24$ is the model constant, $q=(\overline{\mathcal{S}}: \overline{\mathcal{S}}) / 2$, and $r=-\operatorname{det} \overline{\mathcal{S}}$ are the second and third invariants of the rate-of-strain tensor, respectively. The $r^{+}$stands for positive values of $r$ invariant, otherwise $r$ is set to zero.

4.1.3. Wall-adapting local-eddy viscosity model within a variational multiscale framework (VMS-WALE)

Originally formulated for the Smagorinsky model in the Fourier space, the variational multiscale approach considers three classes of scales: large, small and unresolved. If a second filter with filter length $\hat{\ell}$ is introduced, a splitting of the scales can be performed,

$$
f^{\prime}=\bar{f}-\hat{f}
$$

where following Vreman's [30] notation, $f^{\prime}$ is called the small-scale compo-

nent, $\hat{f}$ the large-scale component and $\bar{f}$ is the original resolved quantity. Thus, for the large-scale parts of the resolved $\overline{\boldsymbol{u}}$, a general governing equation can be derived,

$$
\frac{\partial \overline{\mathbf{u}}}{\partial t}+(\overline{\mathbf{u}} \cdot \nabla) \overline{\mathbf{u}}-\nu \nabla^{2} \overline{\mathbf{u}}+\nabla \bar{p}+\overline{\mathcal{F}}=-\nabla \cdot \widehat{\mathcal{T}}-\nabla \cdot \mathcal{T}^{\prime}
$$


In Equation (18) it is possible to identify $\widehat{\mathcal{T}}$ as the subgrid term in the largescale equation and $\mathcal{T}^{\prime}$ as the subgrid small-scale term. Neglecting the effect of unresolved scales in the large-scale equation $(\widehat{\mathcal{T}} \approx 0)$, it is only necessary to model the $\mathcal{T}^{\prime}$. Here the small-small strategy is used in conjunction with the WALE model [17],

$$
\mathcal{T}^{\prime}-\frac{1}{3}\left(\mathcal{T}^{\prime}: \boldsymbol{I}\right) \boldsymbol{I}=-2 \nu_{s g s} \mathcal{S}^{\prime}
$$

where the formulation is closed by means of $\nu_{\text {sgs }}$ obtained with the WALE formulation (see Section 4.1.1). In the present study, the globally conservative adjoint filter proposed by Vreman [30] is used as the second filter with $\hat{\ell}=2 \ell$.

\subsection{Numerical methods for LES}

LES are carried out by using the Termofluids code [31], which is a parallel object-oriented CFD code. The numerical methods used in both momentum and energy equations for LES are essentially the same as the ones detailed in Section 3.2, except for employing second-order conservative schemes on collocated meshes in the discretization of these equations (see for details [32]). As mentioned before, this kind of schemes ensure both stability and conservation of the global kinetic-energy balance on any grid, thus constituting a convenient starting point for LES-like simulations [25]. This numerical methodology has been previously used with accurate results for solving the flow over bluff-bodies with massive separation and turbulent natural convection in enclosures (see for instance $[12,33,34]$ ). 


\subsection{Numerical parameters for LES}

In order to assure that the complex phenomena are reproduced by the LES models, different meshes have been tested. In Table 2 the parameters of three of the tested LES meshes are given. As is done in the DNS, the meshes are generated uniformly in streamwise and spanwise directions, while the hyperbolic-tangent function of Equation (8) is used in the wall-normal direction. Mean flow parameters are obtained by integrating the results during a period of 500 time units in order to assure statistically steady-state solution. The initial transient period of approximately 400 time units is discarded in the light of the information obtained from various probes placed within the domain at locations of different time scales. Results presented in this paper for LES correspond to Mesh C. For further details on the mesh resolution studies see Appendix A.

\begin{tabular}{lcccccc}
\hline Mesh & $N_{x}$ & $N_{y}$ & $N_{z}$ & $N_{\text {tot }} \times 10^{-6}$ & $\gamma_{x}$ & $(\Delta x)_{\min } / H$ \\
\hline Mesh C & 55 & 491 & 16 & 0.43 & 1.97 & $6.0 \times 10^{-4}$ \\
Mesh D & 33 & 319 & 8 & 0.08 & 2.15 & $8.0 \times 10^{-4}$ \\
Mesh E & 55 & 113 & 16 & 0.10 & 1.97 & $6.0 \times 10^{-4}$ \\
\hline
\end{tabular}

Table 2: Main parameters of the numerical simulations for LES. See the caption of Table 1 for details.

\section{Results and discussion}

In this section the results obtained with WALE, QR, and VMS-WALE models are compared in detail with the DNS solution to assess their performance in estimating the turbulent flow under consideration. In order to 
highlight the influence of the models, a solution obtained by the LES code without using any SGS model (No Model) is also presented for Mesh C.

\subsection{Instantaneous flow}

Owing to the buoyancy effects as a consequence of the applied temperature difference at the vertical confining walls, the fluid in contact with the vertical walls moves upwards along the hot wall and downwards along the cold wall. The fluid accelerates along the vertical boundary layers up to a point where the boundary layer is disrupted, thus large unsteady eddies are ejected to the core of the cavity. It is a challenge to capture the location of the transition, which can be affected by the presence of the interacting vertical boundary layers. The extent of the stratified region is directly related with the transition point $[12,19]$, which shifts upstream as the Ra number increases.

In accordance with the results reported by several authors (see $[5,14,19]$, for instance) for similar aspect ratios and $R a$ numbers, a complex flow with laminar, transitional, and turbulence regions is expected. The existence of different flow regimes can be visualized in Figure 6, where time evolution of the dimensionless temperature at different locations is depicted after the initial transient period is washed out (see figure caption for probe locations). Note that the flow in the center of the cavity (P1) is laminar and the temper-

ature value is $\frac{T-T_{c}}{\Delta T_{r e f}}=0.5$, while at the top of the cavity (P3) more chaotic flow is observed. Due to the differences in the temperature fluctuations, a wide range of time scales are expected too.

In Figure 7, a view of the instantaneous isotherms are shown for the DNS. The figure indicates the structure of the flow in the vicinity of the transition 


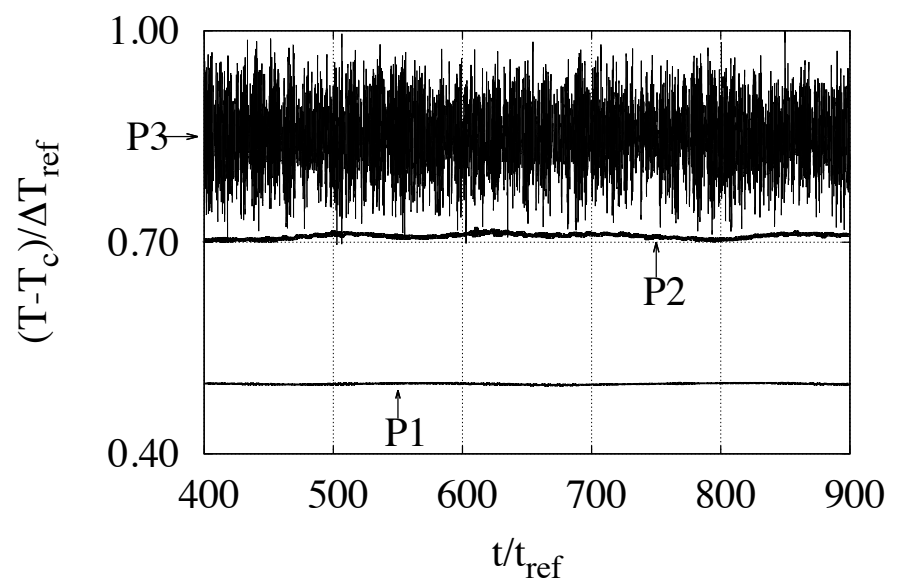

Figure 6: Time evolution of temperature at different domain locations for LES using Mesh C. $\mathrm{P} 1 \equiv(0.1,0.5,0.1), \mathrm{P} 2 \equiv(0.1,0.8,0.1), \mathrm{P} 3 \equiv(0.0024,0.9,0.1)$.

region, near both the hot and cold walls. Away from the upper-left and lower-right corners of the cavity, the flow is stratified with quasi-parallel isosurfaces.

\subsection{Mean flow parameters}

The capability of the models to predict the complex physics of the flow is directly related with their performance in properly capturing the transition location. In order to analyze the transition to turbulence, the local average Nusselt number and its standard deviation are plotted in Figure 8. Note that the standard deviation of the local average Nusselt number is an indicator of the temperature fluctuations. In the laminar regime, fluctuations and thus standard deviations are insignificant. However, at some stage downstream, the standard deviation starts to rise, which points out the onset of turbulence 




(a)

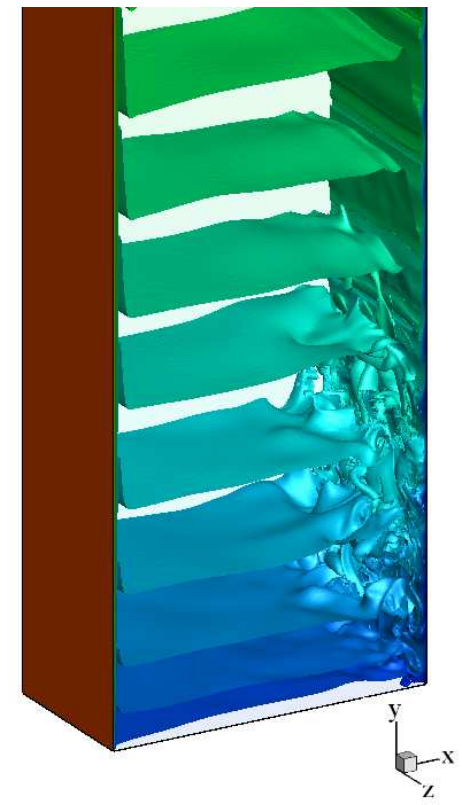

(b)

Figure 7: A view of the instantaneous isotherms for the DNS. Transitional and turbulent regions in the vicinity of hot (left) and cold (right) walls. The axes are shown for orientation.

(see Figure 8(b)). In the No Model and QR results, although in a smaller extent for the latter, the instabilities occur much earlier than in the DNS. On the other hand, for the VMS-WALE and WALE, the onset of turbulence location qualitatively coincides with that of the DNS solution.

In Table 3, the overall averaged Nusselt numbers, defined as $N u=$ $(1 / H) \int_{0}^{H}<N u>d y$, and the parameters related with the transition to turbulence are presented. Note that it is not straightforward to define a clear cut transition to turbulence location, especially in low-level turbulence 


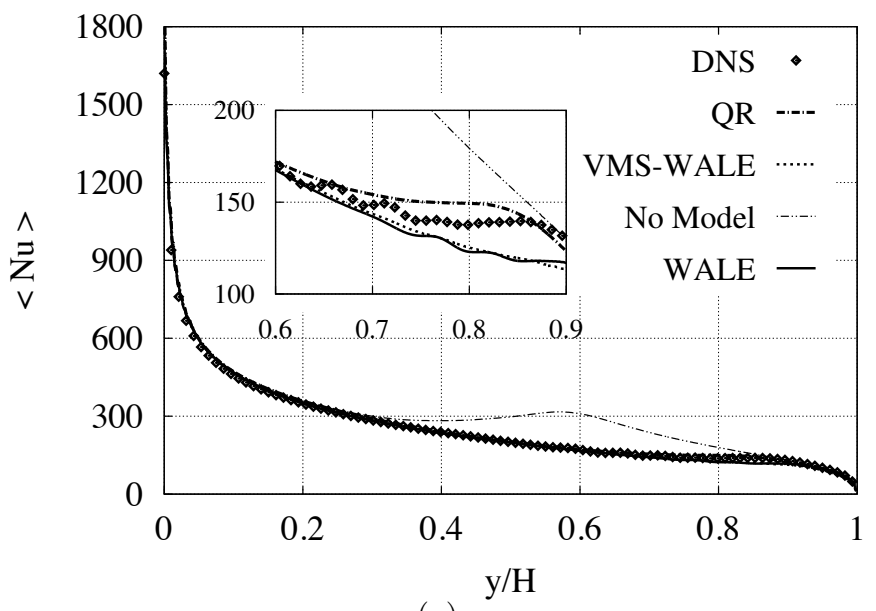

(a)

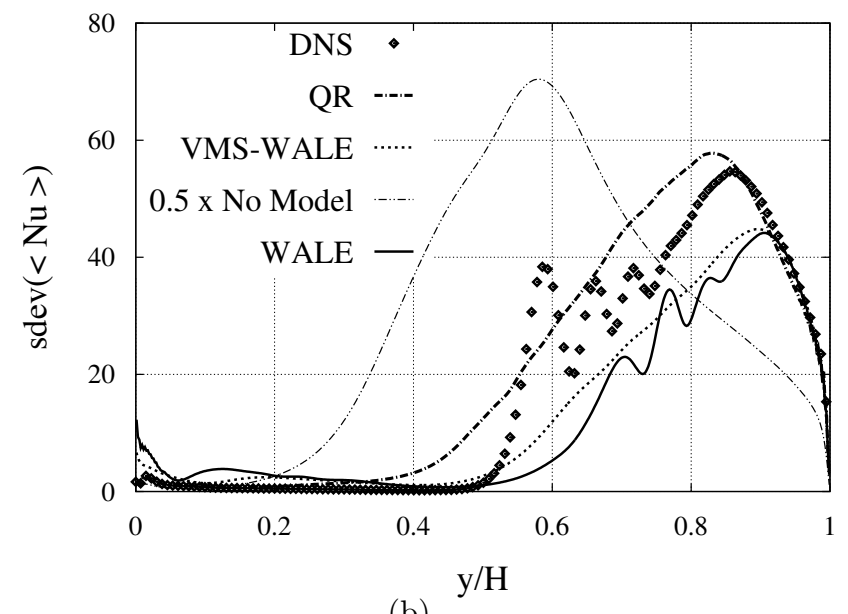

(b)

Figure 8: Local average Nusselt number and its standard deviation along the hot wall for the tested models.

flows. For this reason two locations are presented, the former being the location of the onset of turbulence, $y_{o}$, defined as the the location where the standard deviation of the Nusselt number exceeds $1 \%$ of the local average value. The latter is the maximum fluctuation location, $y_{\max }$, where stan- 
dard deviation of the local average Nusselt number peaks. Regardless of the chosen criterion to define the transition location, the No Model solution indicates upstream transition, $y_{0} / H=0.18$. As for the QR model, the onset of turbulence is predicted slightly upstream $\left(y_{o} / H=0.38\right)$ than the reference DNS solution. Note that the VMS-WALE and WALE models predict better the transition, which occurs around mid height of the cavity. Due to the good achievement of the transition location, the overall averaged Nusselt number is well predicted by these two models within an error of approximately 0.4 $\%$.

\begin{tabular}{lccccc}
\hline & $N u$ & $\mathrm{e}[\%]$ & $y_{o} / H$ & $y_{\max } / H$ & $\mathrm{~S}$ \\
\hline DNS & 256.4 & - & 0.51 & 0.86 & 0.66 \\
QR & 264.0 & 3.0 & 0.38 & 0.83 & 0.68 \\
VMS-WALE & 256.2 & 0.1 & 0.49 & 0.90 & 0.60 \\
No Model & 303.3 & 18.3 & 0.18 & 0.57 & 0.94 \\
WALE & 255.3 & 0.4 & 0.54 & 0.91 & 0.59 \\
\hline
\end{tabular}

Table 3: Overall averaged Nusselt number and transition parameters for the tested models. $y_{o} / H$ and $y_{\max } / H$ are the locations of the onset of turbulence and maximum fluctuation, $\mathrm{S}$ is the thermal stratification in the cavity core, respectively.

As can be observed in the detail of the local average Nusselt number (see Figure 8(a)), the No Model is unable to predict the heat transfer accurately while the models reproduce the DNS results with relatively small discrepancies. This is especially true for the VMS-WALE and WALE models. The WALE model can mimic the complex flow after the onset of turbulence. The standard deviation curve shows that the turbulent fluctuations experience successive reductions and increments, indicating a weak relami- 
narization trend. The WALE is the only model within the tested ones to capture this feature, although this is predicted slightly downstream with respect to the DNS solution. This relaminarization phenomenon seems to be filtered by the VMS-WALE model, although the standard deviation values are quantitatively similar to those of the WALE model.

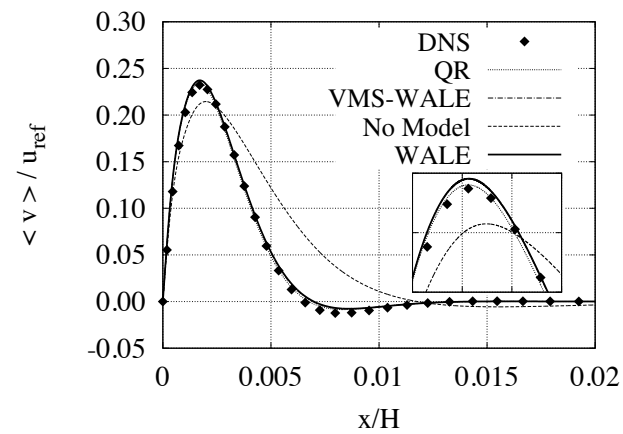

(a)



(b)

Figure 9: Streamwise velocity profile $v / u_{r e f}$ in the vicinity of the hot wall at different locations. (a) $y / H=0.5$ (b) $y / H=0.75$.

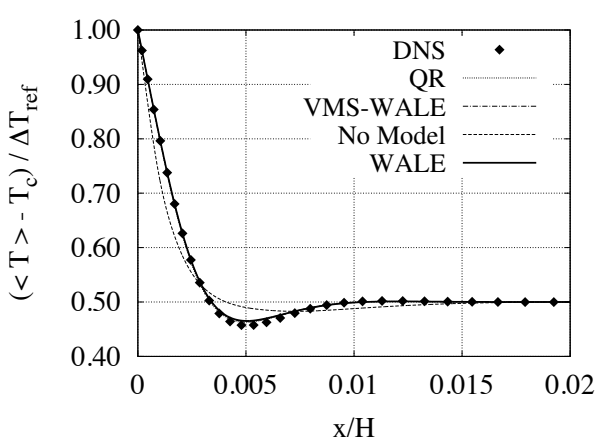

(a)

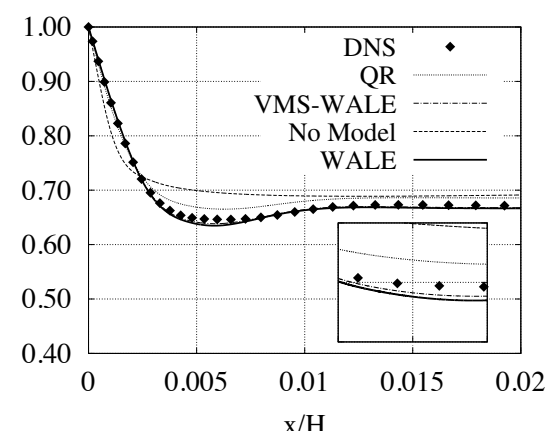

(b)

Figure 10: Temperature profile in the vicinity of the hot wall at different locations. (a) $y / H=0.5$ (b) $y / H=0.75$.

In Figure 9, the streamwise velocity profiles at mid height $(y / H=0.5)$ 
and at $y / H=0.75$ are plotted in the vicinity of the hot wall. Note that the velocity is well predicted by all the models at the mid height. However, as the flow gets downstream, the models are unable to capture the streamwise velocity component with the same precision. The VMS-WALE and WALE overestimate, while the QR underestimates, the maximum streamwise velocity -all within $10 \%$ - performing the QR slightly better than the other models. If no model is used, the discrepancies with the reference solution are of the order of $8 \%$ even at the mid height, where a pronounced thickening of the boundary layer is observed. In the absence of model, for the tested mesh, it seems that the excess of turbulent kinetic energy cannot be dissipated by the molecular viscosity, thus leading to an earlier transition. This implies the deceleration of the fluid due to enhanced wall-normal motion, which justifies the thicker boundary layer. This trend continues at the downstream location, where the physics of the flow can no longer be reproduced as the error is as high as $45 \%$.

The temperature profiles at the same locations are depicted in Figure 10, confirming the high degree of agreement of all the models at mid height. At the downstream location where the flow is more chaotic, the VMS-WALE and WALE models achieve good performance on temperature prediction, whereas the QR model yields a warmer cavity. The warmer cavity prediction of the QR model can be attributed to the upstream transition prediction. In the absence of model, however, the results deviate even qualitatively from the reference solution, which is due to the poor performance in capturing the transition location. It is important to note that both the VMS-WALE and WALE models, in turn, predict qualitatively similar flow structures to those 
of the reference solution, thanks to their refined prediction of the transition location.

The performance of the tested models in the whole cavity can also be assessed by means of the temperature profiles at mid width, $x / W=0.5$, as shown in Figure 11. The conclusions drawn by analyzing the models at particular horizontal profiles (i.e. $y / H=0.5,0.75)$ are seen to be applicable to the entire cavity. The VMS-WALE and WALE models predict the temperature satisfactorily throughout the cavity height, especially between $y / H=0.6$ and $y / H=0.9$ (and the equivalent locations at the bottom of the cavity) where turbulent fluctuations are expected to be influential. The No Model and the QR model, however, overestimate (underestimate) the temperature at the top (bottom) of the cavity. Note that the discrepancies with the DNS solution are more pronounced when no model is used. As for the thermal stratification in the core of the cavity, the No Model highly overestimates the reference value while the model predictions are within approximately $10 \%$.

In Figure 12, the dimensionless time-averaged wall shear stresses obtained are compared with the DNS results. The results indicate that all the tested models can follow the trend of gradual shear stress reduction as observed in the DNS solution. As for the No Model, the marked decrease in the wall shear stress points out the thickening of the boundary layer. Note that the QR model can reproduce the reference wall shear stress values in the top of the cavity, which can also be anticipated from the higher degree of agreement in the streamwise velocity component for this model. 


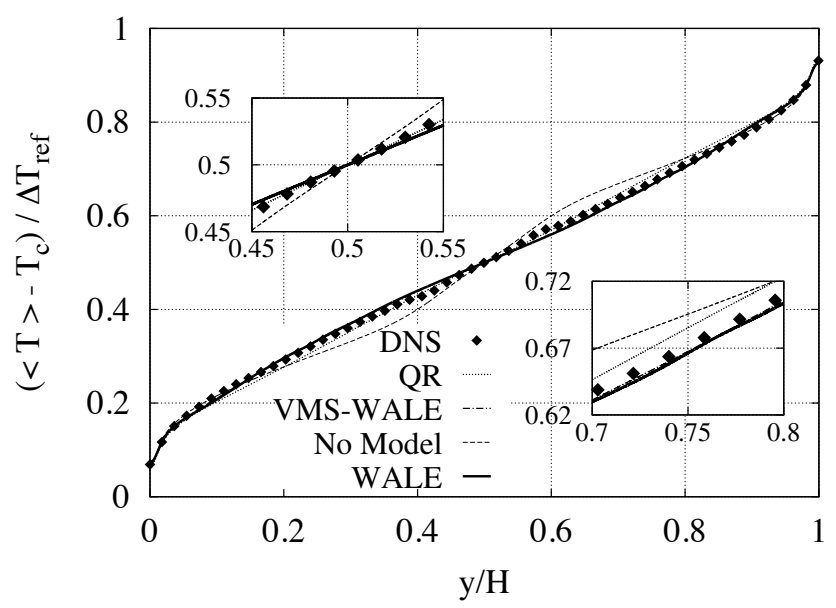

Figure 11: Temperature profiles at mid width along the cavity height.

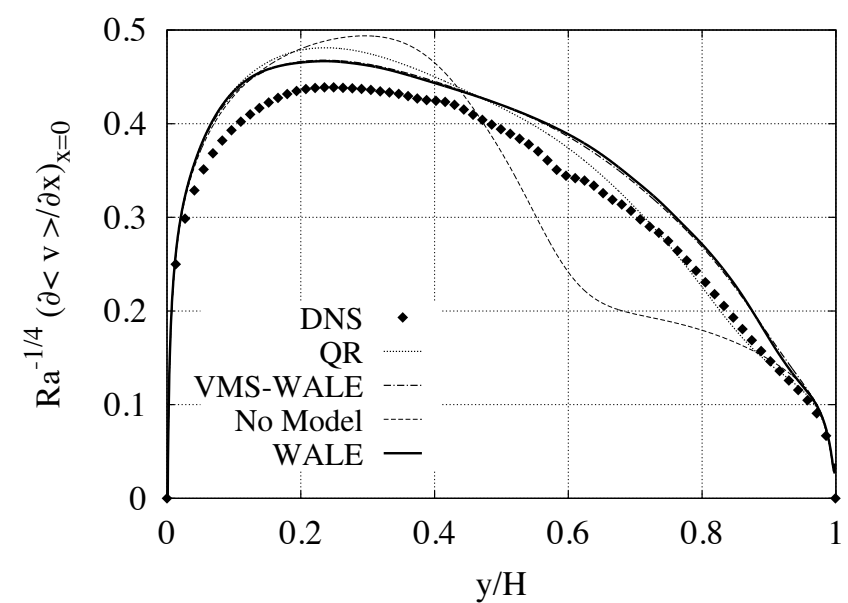

Figure 12: Dimensionless time-averaged wall shear stress at the hot wall scaled by $R a^{1 / 4}$.

\subsection{Turbulent statistics}

The performance of the models in predicting the turbulent statistics is tested at the same locations, i.e. $y / H=0.5$ and $y / H=0.75$. Although 
the No Model has already failed in predicting the mean flow parameters accurately, the corresponding turbulent statistics are also presented for comparison purposes. In Figure 13, the normal and shear Reynolds stresses are depicted. Note that at $y / H=0.5$, the Reynolds stresses for both the No Model and the QR model are significantly greater than the DNS results, which is in accordance with the upstream transition prediction as explained earlier in this section. Moreover, the maxima occur at different locations, indicating different physics of the flow. Note that the Reynolds stresses are overpredicted by the No Model up to three orders of magnitude, showing that the stresses cannot be reproduced even qualitatively without a model. As for the VMS-WALE and WALE models, although the Reynolds stress values are underestimated, the locations of maximum and minimum values are well predicted, with the exception of the local $\left\langle v^{\prime} v^{\prime}\right\rangle$ and $\left\langle u^{\prime} v^{\prime}\right\rangle$ peaks in the boundary layer, as these maxima are predicted slightly closer to the wall. Note that at this location the values are relatively small, indicating quasi-laminar flow. At $y / H=0.75$, all the predictions are of the same order of magnitude, yet the VMS-WALE and WALE perform somehow better than the QR, especially in predicting the dominant $\left\langle v^{\prime} v^{\prime}>\right.$ term.

In Figure 14, the turbulent heat fluxes and the temperature fluctuations are depicted at the same locations. The results confirm the good performance of the VMS-WALE and WALE models, while a similar mismatch in results are observed for the No Model and the QR. Note that the dominant streamwise turbulent heat flux term (see Figure 14(d)) is well predicted by the VMS-WALE and WALE models, not only in magnitude but also in the locations where the maxima occur, which may indicate a satisfactory overall 

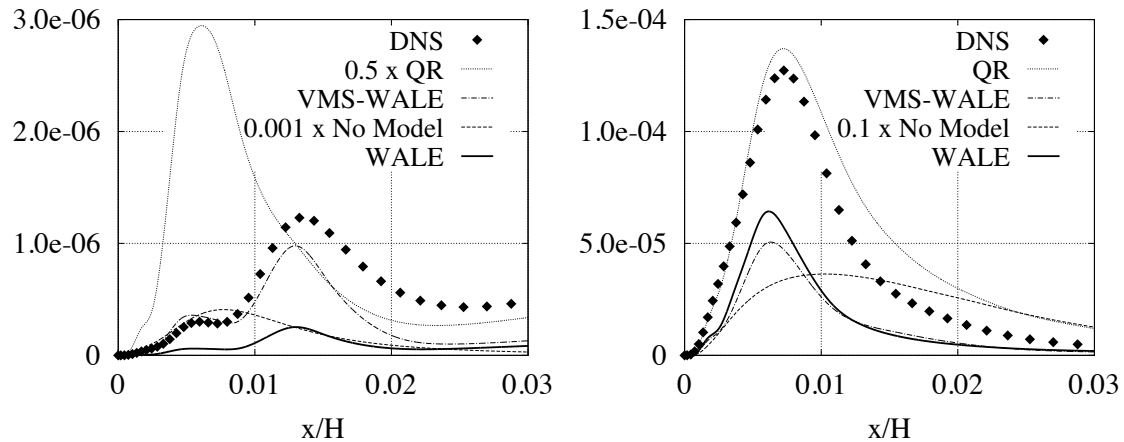

(a) $\left\langle u^{\prime} u^{\prime}>/ u_{\text {ref }}^{2}\right.$ at $y / H=0.5$

(b) $\left\langle u^{\prime} u^{\prime}\right\rangle / u_{\text {ref }}^{2}$ at $y / H=0.75$
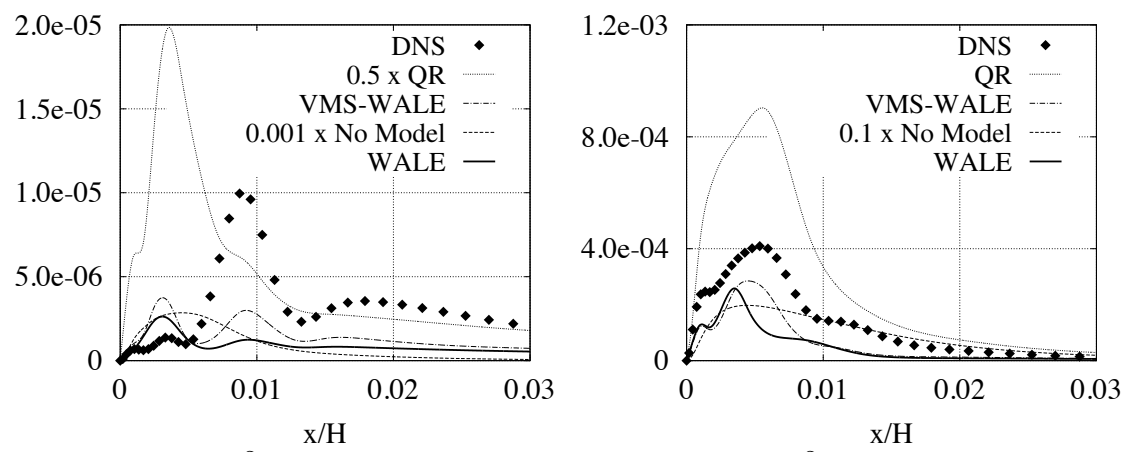

(c) $\left\langle v^{\prime} v^{\prime}>/ u_{\text {ref }}^{2}\right.$ at $y / H=0.5$

(d) $\left\langle v^{\prime} v^{\prime}\right\rangle / u_{\text {ref }}^{2}$ at $y / H=0.75$
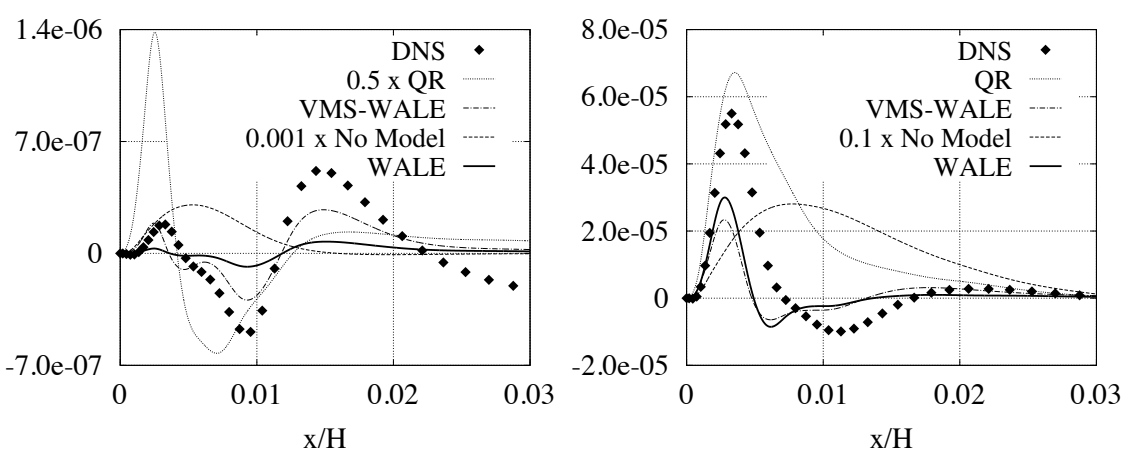

(e) $\left\langle u^{\prime} v^{\prime}>/ u_{\text {ref }}^{2}\right.$ at $y / H=0.5$

(f) $\left\langle u^{\prime} v^{\prime}>/ u_{\text {ref }}^{2}\right.$ at $y / H=0.75$

Figure 13: Reynolds stresses in the vicinity of the hot wall at different locations. (left) $y / H=0.5$ (right) $y / H=0.75$. 
performance of the mentioned models in predicting the flow under consideration.

\section{Conclusions}

The performance of three subgrid-scale models for predicting the turbulent natural convection flow in a water-filled differentially heated cavity of aspect ratio 5 at $R a=3 \times 10^{11}$ and $\operatorname{Pr}=4.31$ has been studied. The models considered are the wall-adapting local-eddy viscosity model (WALE), the QR model, and the WALE model within a variational multiscale framework (VMS-WALE). A direct numerical simulation (DNS) has also been carried out in order to obtain a reference solution. Mean flow parameters and turbulent statistics have been presented for the tested models for their comparison with the DNS solution. It is shown that the performance of the models is directly linked to the accurate prediction of the transition to turbulence in agreement with the observations by other authors.

The results reveal that, for the flow under consideration, although all the models perform well, the VMS-WALE and WALE models perform better due to their capability to capture the transition to turbulence phenomenon, predicting accurately the heat transfer and flow structure. A similar behavior is observed for the turbulent statistics, where the VMS-WALE and WALE generally perform well, reproducing the general trend of the DNS results. It has been also shown that if no SGS model is used, the flow behavior cannot be reproduced even qualitatively due to inaccurate prediction of the transition location.

Finally, it is the aim of the authors to make publicly available the current 


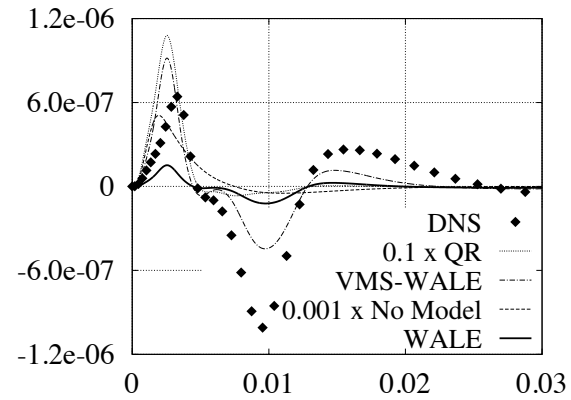

(a) $\left\langle u^{\prime} T^{\prime}>/\left(u_{r e f}^{\mathrm{x} / \mathrm{H}} \Delta T_{r e f}\right)\right.$

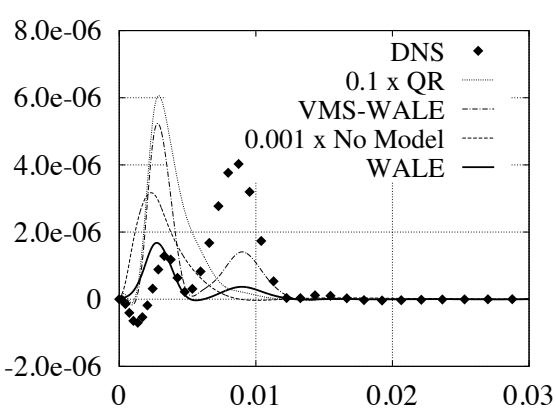

(c) $\left\langle v^{\prime} T^{\prime}>/\left(u_{r e f}^{\mathrm{x} / \mathrm{H}} \Delta T_{r e f}\right)\right.$

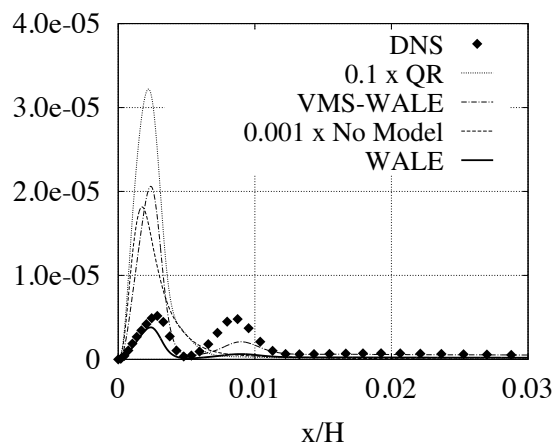

(e) $<T^{\prime} T^{\prime}>/ \Delta T_{r e f}^{2}$



(b) $<u^{\prime} T^{\prime}>/\left(u_{\text {ref }} \Delta T_{\text {ref }}\right)$

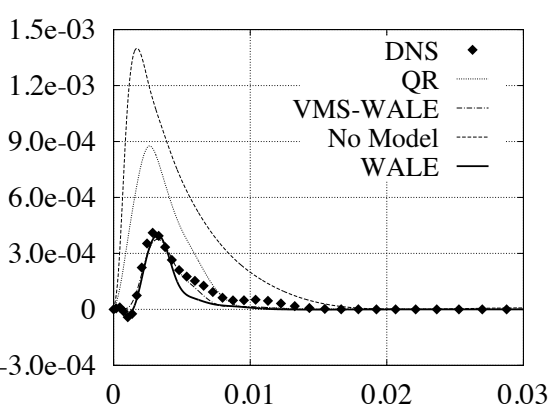

(d) $\left\langle v^{\prime} T^{\prime}>/\left(u_{r e f}^{\mathrm{x} / \mathrm{H}} \Delta T_{r e f}\right)\right.$

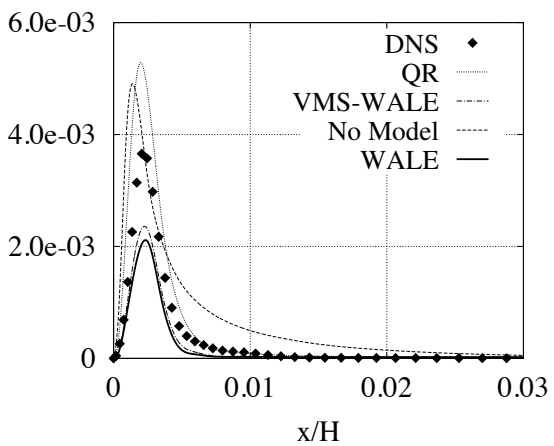

(f) $\left\langle T^{\prime} T^{\prime}>/ \Delta T_{r e f}^{2}\right.$

Figure 14: Turbulent heat fluxes and temperature fluctuations in the vicinity of the hot wall at different locations. (left) $y / H=0.5$ (right) $y / H=0.75$. 
DNS results by creating an extended database [35] which might be useful for validating future RANS and LES studies.

\section{Acknowledgments}

This work has been partially supported by the Ministerio de Economía y Competitividad, Secretaría de Estado de Investigación, Desarrollo e Innovación, Spain (Ref. ENE2010-17801), a Ramón y Cajal postdoctoral contract by the Ministerio de Ciencia e Innovación, Spain (RYC-2012-11996), and the collaboration project between Universitat Politècnica de CatalunyaBarcelonaTech and Termo Fluids S.L. DNS calculations have been performed on the IBM MareNostrum supercomputer at the Barcelona Supercomputing Center. The authors thankfully acknowledge these institutions.

\section{References}

[1] G. De Vahl Davis, Natural convection of air in a square cavity: a benchmark numerical solution, International Journal for Numerical Methods in Fluids 3 (1983) 249-264.

[2] R. Henkes, F. van Der Vlugt, C. Hoogendoorn, Natural convection flow in a square cavity calculated with low Reynolds-number turbulence models, International Journal of Heat and Mass Transfer 34 (1991) 377-388.

[3] T. Fusegi, J. M. Hyun, K. Kuwahara, B. Farouk, A numerical study of three-dimensional natural convection in a differentially heated cubical enclosure, International Journal of Heat and Fluid Flow 34 (6) (1991) $1543-1557$. 
[4] R. Janssen, R. Henkes, Accuracy of finite-volume discretizations for the bifurcating natural-convection flow in a square cavity, Numerical Heat Transfer, Part B 24 (1993) 191-207.

[5] P. Le Quéré, M. Behnia, From onset of unsteadiness to chaos in a differentially heated square cavity, Journal of Fluid Mechanics 359 (1998) 81-107.

[6] S. Xin, P. Le Quéré, Direct numerical simulations of two-dimensional chaotic natural convection in a differentially heated cavity of aspect ratio 4, Journal of Fluid Mechanics 304 (1995) 87-118.

[7] F. X. Trias, M. Soria, A. Oliva, C. D. Pérez-Segarra, Direct numerical simulations of two and three dimensional turbulent natural convection flows in a differentially heated cavity of aspect ratio 4, Journal of Fluid Mechanics 586 (2007) 259-293.

[8] F. X. Trias, A. Gorobets, M. Soria, A. Oliva, Direct numerical simulation of a differentially heated cavity of aspect ratio 4 with Rayleigh numbers up to $10^{11}$ - Part II: Heat transfer and flow dynamics, International Journal of Heat and Mass Transfer 53 (2010) 674-683.

[9] J. Patterson, J. Imberger, Unsteady natural convection in a rectangular cavity, Journal of Fluid Mechanics 100 (1980) 65-86.

[10] P. Le Quéré, Transition to unsteady natural convection in a tall waterfilled cavity, Physics of Fluids A 2 (1990) 503-515.

[11] P. J. A. Janssen, R. A. W. M. Henkes, Influence of Prandtl number on 
instability mechanisms and transition in a differentially heated square cavity, Journal of Fluid Mechanics 290 (1995) 319-344.

[12] D. Kizildag, I. Rodríguez, A. Oliva, O. Lehmkuhl, Limits of the Oberbeck-Boussinesq approximation in a tall differentially heated cavity filled with water, International Journal of Heat and Mass Transfer 68 (2014) 489-499.

[13] S. Peng, L. Davidson, Large eddy simulation for turbulent buoyant flow in a confined cavity, International Journal of Heat and Fluid Flow 22 (2001) 323-331.

[14] D. Barhaghi, L. Davidson, Natural convection boundary layer in a 5:1 cavity, Physics of Fluids 19 (2007) 125106.

[15] J. Smagorinsky, General circulation experiments with the primitive equations: I. The basic experiment, Monthly Weather Review 91 (1963) 99-164.

[16] M. Germano, U. Piomelli, P. Moin, W. Cabot, A dynamic subgrid-scale eddy viscosity model, Physics of Fluids A 3 (1991) 1760.

[17] F. Nicoud, F. Ducros, Subgrid-scale stress modeling based on the square of the velocity gradient tensor, Flow, Turbulence and Combustion 62 (1999) 183-200.

[18] G. E. Lau, G. H. Yeoh, V. Timchenko, J. A. Reizes, Large-eddy simulation of turbulent buoyancy-driven flow in a rectangular cavity, International Journal of Heat and Fluid Flow 39 (2013) 28-41. 
[19] F. X. Trias, A. Gorobets, C. D. Pérez-Segarra, A. Oliva, DNS and regularization modeling of a turbulent differentially heated cavity of aspect ratio 5, International Journal of Heat and Mass Transfer 57 (2013) 171182.

[20] N. S. Ghaisas, D. A. Shetty, S. H. Frankel, Large eddy simulation of thermal driven cavity: Evaluation of subgrid-scale models and flow physics, International Journal of Heat and Mass Transfer 55 (2013) 606-624.

[21] A. Sergent, P. Joubert, S. Xin, P. Le Quéré, Resolving the stratification discrepancy of turbulent natural convection in differentially heated airfilled cavities Part II: End walls effects using large eddy simulation, International Journal of Heat and Fluid Flow 39 (2013) 15-27.

[22] R. Verstappen, When does the viscosity damp subfilter scales sufficiently?, Journal of Scientific Computing 49 (1) (2011) 94-110.

[23] T .J .R. Hughes, L. Mazzei, K. E. Hanzen, Large eddy simulation and the variational multiscale method, Computing and Visualization in Science 3 (1) (2000) 47-59.

[24] R. Cheesewright, K. J. King, S. Ziai, Experimental data for the validation of computer codes for the prediction of two-dimensional buoyant cavity flows, in: Proceedings of Significant Questions in Buoyancy Affected Enclosure or Cavity Flows, ASME, Heat Transfer Division, HTD, vol 60 (1986) 75-81.

[25] R. W. C. P. Verstappen, A. E. P. Veldman, Symmetry-preserving 
discretization of turbulent flow, Journal of Engineering Mathematics 187 (1) (2003) 343-368.

[26] F. X. Trias, O. Lehmkuhl, A self-adaptive strategy for the time integration of Navier-Stokes equations, Numerical Heat Transfer, Part B 60 (2) (2011) 116-134.

[27] P. J. Roache, Code verification by the method of the manufactured solutions, Journal of Fluids Engineering 124(1) (2001) 4-10. doi:10.1115/1.1436090.

[28] J. Fröhlich, C. P. Mellen, W. Rodi, L. Temmerman, M. A. Leschziner, Highly resolved large-eddy simulation of separated flow in a channel with streamwise periodic constrictions, Journal of Fluid Mechanics, 526, 1966 (2005), doi: 10.1017/S0022112004002812.

[29] P. Sagaut, Large eddy simulation for incompressible flows, SpringerVerlag, 2006.

[30] A. Vreman, The adjoint filter operator in large-eddy simulation of turbulent flow, Physics of Fluids 16 (6) (2004) 2012, doi: 10.1063/1.1710479.

[31] O. Lehmkuhl, C. D. Pérez-Segarra, R. Borrell, M. Soria, A. Oliva, TERMOFLUIDS: A new Parallel unstructured CFD code for the simulation of turbulent industrial problems on low cost PC cluster, in: Proceedings of the Parallel CFD 2007 Conference, 2007, pp. 1-8.

[32] L. Jofre, O. Lehmkuhl, J. Ventosa, F. X. Trias, A. Oliva, Conservation properties of unstructured finite-volume mesh schemes for the Navier- 
Stokes equations, Numerical Heat Transfer, Part B 65 (1) (2014) 53-79, doi:10.1080/10407790.2013.836335.

[33] I. Rodríguez, R. Borrell, O. Lehmkuhl, C. D. Pérez-Segarra, A. Oliva, Direct numerical simulation of the flow over a sphere at $R e=3700$, Journal of Fluid Mechanics 679 (2011) 263-287.

[34] O. Lehmkuhl, I. Rodríguez, A. Baez, A. Oliva, C. D. Pérez-Segarra, On the large-eddy simulations for the flow around aerodynamic proles using unstructured grids, Computers and Fluids 84 (2013) 176-189.

[35] The DNS data presented in this article are available by contacting the authors.

\section{Appendix A. Mesh refinement studies for LES}

The success of the numerical simulations for the configuration under investigation is strongly dependent on the mesh resolution, since the flow has regions with different flow regimes, thus with time scales of different orders of magnitude. Different meshes have been tested in order to search for a good compromise between the accuracy and cost of the simulations, attaining the criteria of sufficient resolution in the boundary layer and the core of the cavity. Moreover, it has been observed that the streamwise resolution is particularly critical in order to capture the transition to turbulent location. The present investigation has shown that the transition phenomenon cannot be properly detected unless a moderately high streamwise resolution is used. Consequently, for the flow under consideration, the reduction in the 
grid with respect to the DNS solution is mainly in the wall-normal and spanwise directions, which are observed to be less critical in comparison with the streamwise direction.

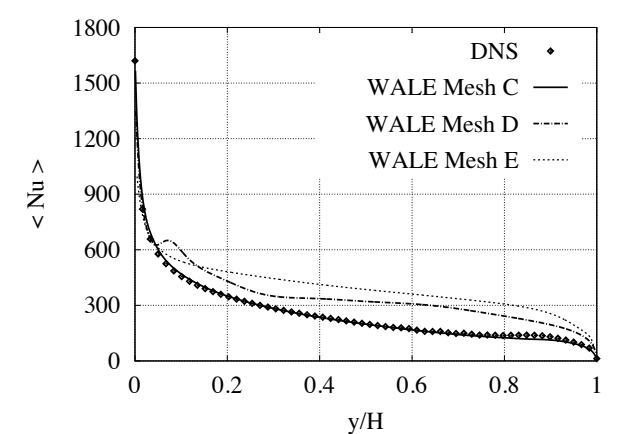

(a)

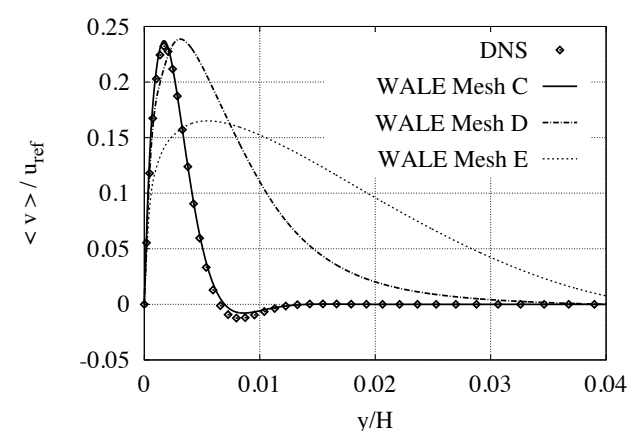

(b)

Figure A.15: Mesh refinement studies. (a) Local average Nusselt number (b) Streamwise velocity at mid height.

In Figure A.15, the local average Nusselt number and streamwise velocity component are depicted for two coarse meshes (Mesh D and Mesh E) and a fine mesh (Mesh C) using WALE model (see Table 2 for details). The results are compared with the DNS data. Note that the Mesh D and Mesh $\mathrm{E}$ are at least 4 times coarser than the Mesh C, which in turn is about 46 times coarser than the DNS mesh as the total number of CVs are concerned. It can be observed that the coarse mesh solutions highly overestimate the overall averaged Nusselt number due to very early transition to turbulence. Consequently, this causes a pronounced thickening in the boundary layer at mid height of the cavity. This is especially the case for the Mesh E which, despite having identical resolution to the fine mesh in wall-normal and spanwise directions, provides the poorest results due to insufficient resolution in the streamwise direction, thus indicating the influence of this direction in the 
model performance. Nevertheless, the Mesh C is capable of reproducing the time averaged results satisfactorily, due primarily to its performance in capturing the transition. Owing to the observed accuracy in the performed mesh refinement studies, it has been concluded that the Mesh $\mathrm{C}$ is an adequate mesh for obtaining results by means of LES for the present configuration. 